valent cations. The three-dimensional tweedy modulation, with components along the $\langle 110\rangle$ and [001] directions, has been systematically studied by transmission electron microscopy in $\mathrm{YBa}_{2}\left(\mathrm{Cu}_{1-\mathrm{x}} \mathrm{M}_{\mathrm{x}}\right)_{3} \mathrm{O}_{7-8}(\mathrm{M}=\mathrm{Fe}, \mathrm{Co}$ or $\mathrm{Al} ; \mathrm{x}>0.025)$. We observed the streaking and splitting of both fundamental and satellite diffraction spots in selected-area diffraction mode and nanodiffraction mode, respectively. Careful analysis of the diffuse scattering suggests that the $<110\rangle$ modulation can be attributed to pseudo-periodic domains with $\langle 110\rangle$ twinning displacement, while the [001] modulation reflects the stacking of such domains on top each other along the c-axis. The size of the domain (or the period of the modulation) decreases with increasing dopant concentration. Intensity calculations of the tweed image and diffuse scattering using a displacement wave model and Monte Carlo simulation show good agreement with the observations. The presence of off-stoichiometric oxygen, or variation in local orthorhombicity, was found to be responsible for such structural modulation.

Discussions and collaborations with Z.X. Cai, J. Tafto, M. Suenaga, and J.M. Cowley are gratefully acknowledged. This research was supported by U.S. Department of Energy under contract DE-AC02-76H00016.

\section{MS-13.01.05 COHERENT CONVERGENT-BEAM ELECTRON DIFFRACTION}

M.Terauchi, K.Tsuda, M.Tanaka, T.Kaneyama* and T.Honda* Res. Inst. Sci. . Meas., Tohoku Univ., Japan, *JEOL Ltd., Japan.

Convergent-Beam Electron Diffraction (CBED) is a powerful technique to identify the symmetries of crystals and the characters of lattice defects. The explanations of the experimental results in these cases are based on the assumption that incident electrons falling on a specimen are incoherent. When a high coherent elecfalling on a specimen are incoherent. When a high coherent elecfringes are produced in the overlapping regions of CBED disks. Lin and Cowley (Ultramicroscopy, 19(1986)31) observed the interference fringes using a STEM microscope, though the fringes were distorted by the aberration of the probe forming lens. Bristol group (Ultramicroscopy, 41(1992)423) obtained the interference fringes of a lattice spacing $15 \AA$ of $6 \mathrm{H}-\mathrm{SiC}$ without the distortion using a new TEM microscope. The interference fringes showed a shift of a half period due to a glide plane. The resolution of the fringes $15 \AA$ is rather large for applying this technique to other materials. We have obtained similar interference fringes of smaller lattice spacings of $\mathrm{FeS}_{2}, \mathrm{Sr}_{2} \mathrm{Nb}_{2} \mathrm{O}_{7}, \mathrm{Sr}_{2} \mathrm{Ta}_{2} \mathrm{O}_{7}, \mathrm{La}_{2} \mathrm{Ti}_{2} \mathrm{O}_{7}, \mathrm{Mo}_{8} \mathrm{O}_{23}$ and $\mathrm{TiO}_{2}$ (Brookite) using electron microscopes JEM2010F and JEM100CX-FEG equipped with a FEG.

The minimum lattice spacing, from which we could obtain the interference fringes (right figure), is $2.7 \AA$ or the lattice spacing of the 200 reflection of $\mathrm{FeS}_{2}$. The vertical fringes $A$ corresponding to the spacing $5.4 \AA$ are produced by the interference between the 021 and the 020 reflec tions. The horizontal fringes $B$ corresponding to the spacing $2.7 \AA$ are due to the interference between the 002 and the 022 reflections.
MS-13.01.06 HIGH SPATIAL RESOLUTION OF DIFFRACTION INFORMATION IN LARGE-ANGLE CONVERGENT-BEAM ELECTRON DIFFRACTION PATTERNS FROM CROSSSECTIONAL SPECIMENS OF $\mathrm{Ge}_{x} \mathrm{Si}_{1 \cdot x} / \mathrm{Si}$ STRAINED-LAYER SUPERLATTICES By X.F. Duan ${ }^{*, k, s}$, D. Chern $s^{s}$ and J.W. Steeds $s^{s}$

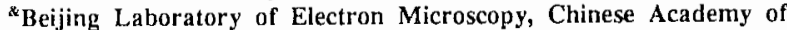
Sciences, P.O. Box 2724, 100080 Beijing, P.R. China. ${ }^{5}$ H.H. Wills Physics Laboratory, University of Bristol, Bristol BS8 1TL, U.K.

Fine diffraction lines in large-angle convergent-beam electron diffraction (LACBED) patterns can be used to investigate the strain in a lattice misfit system because they are sensitively dependent on small changes of the spacing between lattice planes. Since the probe is slightly defocussed, equivalent to imaging the specimen with a convergent spherical wave, a LACBED pattern formed in the diffraction plane of the microscope is superimposed on a high spacial resolution shadow-image of the specimen. In the case of cross-sectional specimens of $\mathrm{Ge}_{4} \mathrm{Si}_{1} / \mathrm{Si}$ strained-layer superlattices (SLS) LACBED can give much information on local strain and misfit stress relaxation. It is important that the probe size, which defines the spatial resolution of the shadow-image, should be as small as possible. The elastic relaxation of strain in the very thin samples used for cross-sectional transmission electron microscopy (XTEM) must be taken into account. The diffraction lines in the GeSi layers are shifted from their position in the Si layers by amount determined by the misfit strain and the subsequent relaxation. We shall demonstrate that the line shifts in XTEM specimen caused by the effects of misfit strain and stress relaxation can be separated. This technique can therefore be used to profile strain and misfit stress relaxation in SLS.

PS-13.01.07 THEORY OF SPIRAL CRYSTALLOGRAPHY bY FAN XuDong school of physics, University of Melbourne, Earkville, VIC 3052, Australia.

Spiral structures exist widely in nature; in both biological and inorganical materials. One of the most perfect examples occurs in botanical species where the structures exhibit alternating visible spirals known as parastichies. The latter always have numbers given by a Fibonacci series. Apart from their present day genetic origin, these types of spiral lattices were also obtained by close packing of growing discs, just as the hexagonal pattern can be obtained by close packing of equal size discs (see Bursill and Fan, Mod. Phys. Letts. B2, 1245-1252(1988)

In this paper a generalized abstract model is developed based on the mechanism of the close-packing model, to erable the critical role played by the initial

conditions and the growth law to be analyzed. This model describes the growing disc by a one-dimensional single peak centro-symmet $x$ ical function $f(x)$. The spiral lattice aggregates are thus generalized as a multi peak function $F_{n}(x)$ obtained by adding $f(x)$ at the global minimum of the existing aggregate $F_{n-2}(x)$. Both functions $f(x)$ and $F(x)$ have the sa porlodicity, reflecting the angular divergens paranetaren the splral lattice. The pexlodicty may be taken as 1 , with no loss of generality. The divergence interval, defined

Similar to the divergence angle of a spixal lattice aggregate, the generalized divergence interval mean This result agrees of course with our earlier graphical computer simulations.

A kinematic approach is attempred and its corresponding initial conditions investigated a kinematic process of initial conditions investigated. A kinematic proce this model is where chere is a ways a sel for each stage contiguration of the aggregate functions and the conseguent divergence intervals can be expressed with consequent divergence intervals can be expressed with recurreme formulae. Using the latter, the propert tound to converge, with limits simply related to the golden mean

Much effort was devoted to searcin analytically for a Eunctional form appropriate for representing the initial conditions in as gereral a way as possible at this stage, the initial conditions, required fo: convergence, have been found within certain limits. 